\title{
Cobalt Disilicide/Silicon(001) Interfaces
}

\author{
A. Bleloch, M. Falke, and U. Falke, \\ SuperSTEM Laboratory, CLRC, Daresbury WA4 4AD, UK.
}

It is the mark of a significant breakthrough when an experimental technique as mature as electron microscopy together with a materials system as well studied as metal silicide/silicon interfaces reveals structures that had not been suspected to exist. High angle annular dark field (HAADF) imaging in aberration corrected scanning transmission electron microscope (STEM) instruments has demonstrated resolution better than $0.1 \mathrm{~nm}$ at $100 \mathrm{kV}$ and has been used to solve a variety of materials problems. Here we describe progress on the study of the cobalt disilicide/silicon(001) interface. First the defect-free interfaces are described followed by images of what the authors believe to be hitherto unreported and still incompletely characterised interface defects.

$\mathrm{CoSi}_{2}$ has the $\mathrm{CaF}_{2}$ structure $(\mathrm{Fm} 3 \mathrm{~m})$, which is cubic and slightly smaller than the diamond structure of $\mathrm{Si}(\mathrm{Fd} 3 \mathrm{~m})$ - the lattice mismatch is $-1.2 \%$. This silicide is a good conductor that also allows atomically smooth conductor/ semiconductor junctions to be generated by epitaxial growth on silicon. Buried films 57nm thick were grown using the Allotaxy method [1]. Cross-sectional samples were then prepared by tripod polishing and brief low angle ion milling.

Two interface structures have been observed to exist. The first and most common was unequivocally observed for the first time by HAADF imaging in an aberration corrected STEM, initially for $\mathrm{NiSi}_{2}$ [2] and then also for $\mathrm{CoSi}_{2}$ [3]. This structure shown in Fig 1 is a $2 \times 1$ reconstructed interface containing a 7-fold coordinated Co atom (the so called "7-fold R" $\left(\mathrm{R}=\right.$ relaxed) model [4]). The second structure observed less often in $\mathrm{CoSi}_{2}$ and not at all in $\mathrm{NiSi}_{2}$ is a Si rich 1x1 structure containing 8-fold coordinated Co. The observed interfaces are consistent with first principle total energy calculations as demonstrated by the data in Table 1[4].

At this interface, dislocations are expected for two reasons. First, misfit dislocations at a commensurate interface would be required for this film geometry. Second, a dislocation is required at interface domain boundaries. This is due to the different crystal symmetry of the silicide compared to silicon. Figure 1 shows an example of the extended and complex defect that is associated with a boundary between a $2 \times 1$ region and a $1 \times 2$ region in the 7 -fold $\mathrm{R}$ structure. Two layers of a so-called b-type Si twin can be seen in addition to a complex rearrangement of the interface structure at each edge of the extended defect. This re-arrangement is the subject of continuing study. However, we can see by counting atomic planes that the structure includes a dislocation made up of both a (110) $\mathrm{CoSi}_{2}$ plane $\left(\boldsymbol{b}_{1}=1 / 4[110]\right)$ and an (001) plane incorporated in the $(2 \times 1)$ interface reconstruction $\left(\boldsymbol{b}_{2}=1 / 4[001]\right)$ resulting in the necessary Burgers vector $\boldsymbol{b}=1 / 4[1101]$ required between the $2 \times 1$ and $1 \times 2$ domains.

Figure 2 shows another defect structure that could be described as an additional reconstruction of the 8 -fold interface structure in which every 5 th atomic column at the interface appears to have the first Co and a Si column swapped. This structure is still under investigation. 
Table 1 Data from Yu et al [4] - calculated interface energies relative to the 7fold R model. Energies are in $\mathrm{eV}$ per atom in increasing order for $\mathrm{NiSi}_{2}$. (bold $=$ observed observed)

\begin{tabular}{|l|l|l|}
\hline Interface model & $\mathrm{NiSi}_{2}$ & $\mathrm{CoSi}_{2}$ \\
\hline 7-fold $R(2 x 1)$ & $\mathbf{0 . 0 0}$ & $\mathbf{0 . 0 0}$ \\
\hline Sixfold & 0.42 & 0.87 \\
\hline 7-fold U & 1.52 & 1.01 \\
\hline 8-fold & 1.74 & $\mathbf{0 . 2 7}$ \\
\hline BJV 2x1 compositional & 2.76 & 1.92 \\
\hline 8-fold/dimer (LJY) & unstable \\
\hline
\end{tabular}

Fig. 1. $\mathrm{CoSi}_{2}$ above with Si below. Looking down the $\mathrm{Si}$ [110] pole with the (001) direction vertical. Two views of the 7-fold $\mathrm{R}$ interface atoms are superimposed [5].

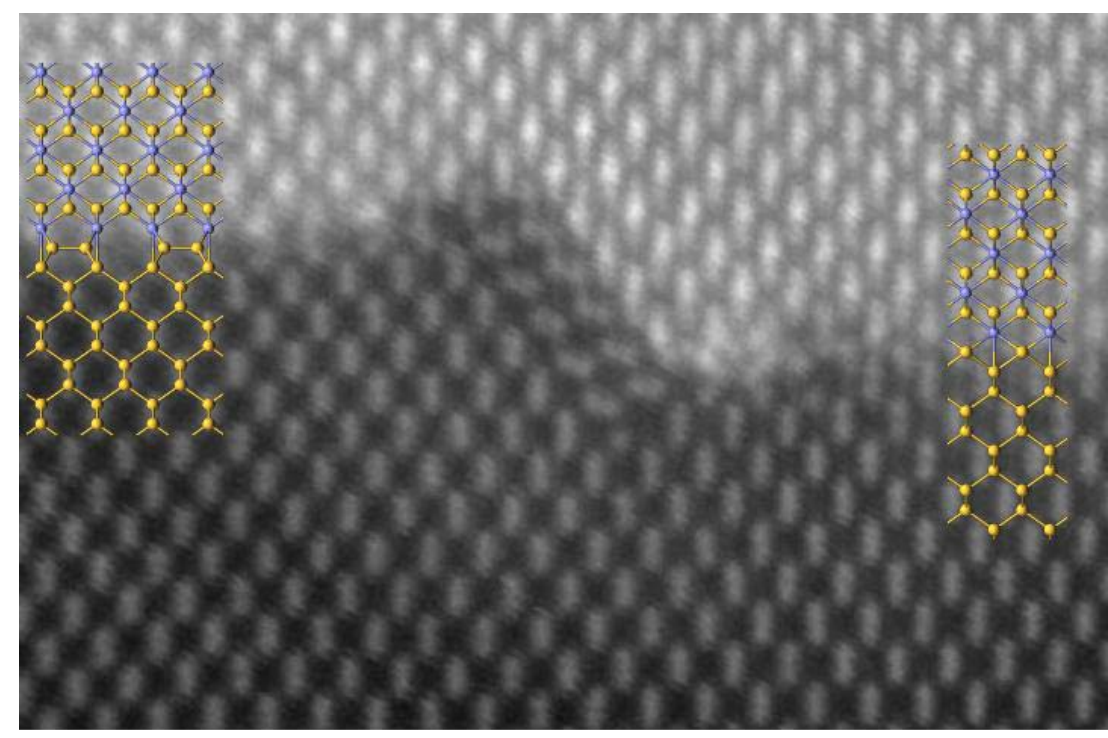

Fig. 2. 8-fold 1x1 interface with every fifth Co column swapped with a Si column (indicated with arrows) [5]

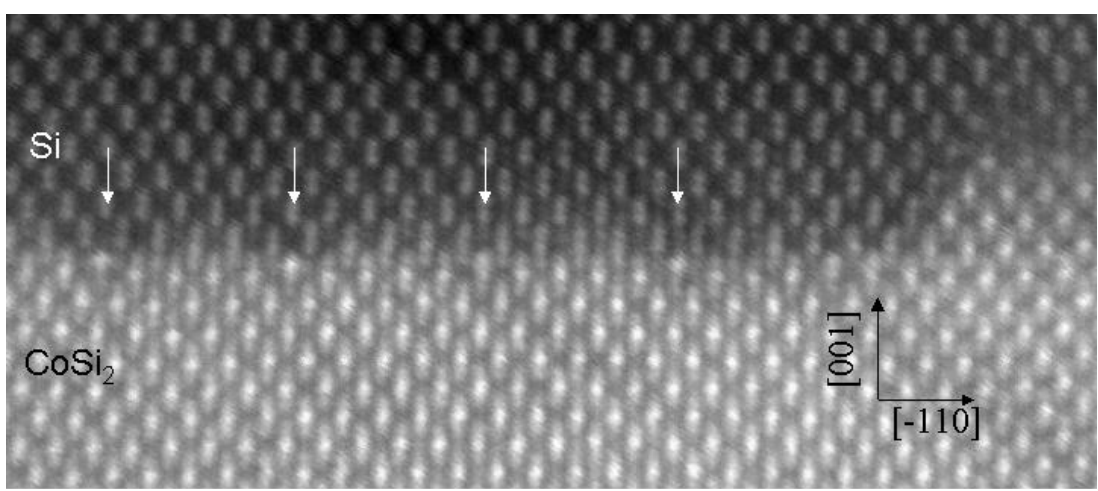

References and acknowledgements:

1. S. Mantl, H.L. Bay, Appl. Phys. Lett. 61, 267 (1992)

2. U. Falke, A. Bleloch, M. Falke, St. Teichert, Phys.Rev.Lett.92, 116103 (2004)

3. M. Falke et al submitted to Applied Physics Letters

4. B. D. Yu etal. J. Vac. Sci.Technol. B 19, 1180 (2001)

5 Sample from St. Teichert, G. Beddies, H.-J. Hinneberg, Insitute of Physics, TU-Chemnitz 6 We would like to thank the EPSRC, CLRC Daresbury, EMAG (IOP), and the RMS 